\title{
Ionic liquids accelerating cycloaddition between 1-aryl-2-halocyclopropenes and furan
}

\author{
May-Fan Ding, Shaw-Tao Lin,* and Woan-Ju Chang \\ Department of Applied Chemistry; Providence University, Sha-Lu, Taichung, Taiwan, 43301 \\ E-mail:sdlin@pu.edu.tw
}

\begin{abstract}
Treatment of a series of 1-aryl-2,2-dihalocyclopropanes with $t$-BuOK at $-10{ }^{\circ} \mathrm{C}$ gave the corresponding 1-aryl-2halocyclopropenes, which react with furan in a RTIL to give a fair good yield of the [4+2]-cycloadducts with more than $90 \%$ of the exo-isomer. The imidazolium type ionic liquids are able to accelerate this cycloaddition process with high steric selectivity. Neither pyrrole nor thiophene undergoes the cycloaddition with cyclopropene to form the [4+2]-cycloadduct. 1-Aryl-3,3-difluoro-2-halocyclopropenes are inert towards furan even at a temperature higher than $100{ }^{\circ} \mathrm{C}$.
\end{abstract}

Keywords: RTIL, cyclopropenes, cycloaddition, exo-adduct, simulation

\section{Introduction}

Cyclopropene has attracted the attention of both theoretical and experimental chemists due to its special place as the simplest small ring with a double bond. ${ }^{1}$ It contains $27.7 \mathrm{Kcal} / \mathrm{mol}$ of olefinic strain energy and $55.2 \mathrm{Kcal} / \mathrm{mol}$ of strain energy ${ }^{1}$ with a short double bond of $1.296 \AA^{2}$; therefore it is expected to be a highly reactive molecule. Unsubstituted cyclopropene 1 is a potentially explosive gas and is oligomerizing rapidly via ene reactions. ${ }^{3}$ In contrast to $\mathbf{1}$, the substituted cyclopropenes, which are stabilized by the substituent, are stable enough to be utilized as a dienophile. The cycloaddition of cyclopropenes have been studied in some extent. ${ }^{4}$ The reactivity of dienes toward cyclopropene and the steric selectivity strongly depend on the nature of the diene and the substituent on the cyclopropene. Although cycloadditions using heterocycles as diene have been widely applied to prepare some potential pharmaceutical derivatives, ${ }^{5}$ the cycloaddition between phenylcyclopropene and furan is less successful. ${ }^{\text {5a }}$

Room-temperature ionic liquids (RTIL) provide a solvent environment that is quite different from any other available solvent at room temperature. With their unique character, the RTIL may induce solvent effects on a wide range of processes. A chloroloaluminate ionic liquid is able to reverse the steric selectivity in the reaction of cyclopentadiene with methyl methacrylate from an exo-adduct in a common organic solvent to an endo-adduct. ${ }^{6}$ Such a phenomenon is also observed in the reaction of cyclopentadiene with ethyl acrylate in an imidazolium system. ${ }^{7}$ This may be rationalized by the "polarity" of the RTIL which is able to stabilize the more polar (endo) activated complex. ${ }^{8}$ Herein, we like to report the cycloaddition of 1-aryl2-halocyclopropenes ( $\mathrm{X}=\mathrm{Br} 2, \mathrm{Cl}$ 3) and furan, pyrrole or thiophene effected by a RTIL.

\section{Results and Discussion}

Compound 2 always decomposes to form either allene or acetylene in the presence of either strong base or at elevated reaction temperature. ${ }^{9}$ 1-Bromo-2-phenylcyclopropene has been prepared from the reaction of 1-phenyl-1,2,2-tribromocyclopropane and $\mathrm{MeLi}$ at $-45^{\circ} \mathrm{C}$ or even lower to avoid the ring opening reaction. ${ }^{5}$ In this study, compounds 2,3 were prepared from the reaction of 1-aryl-2,2-dihalocyclopropanes with $t$-BuOK in hexane at $-10{ }^{\circ} \mathrm{C}$ for $4 \mathrm{~h}$. (Scheme 1) The formation of 2,3 is monitored using GC-MS analysis. (Figure 1). Compounds 2, 3 prepared from this reaction are used without purification. The reactions of compound 2,3 and a large excess of furan $(1: 10)$ were conducted within a sealed system at $30{ }^{\circ} \mathrm{C}$ for $12 \mathrm{~h}$ due to the thermal instability of cyclopropenes and the lower boiling point of furan (bp. $31-33^{\circ} \mathrm{C}$ ). Flash chromatography afforded a mixture and the ratio of exo- and endo-isomer was estimated according to the peak areas obtained from the GC- MS analysis. 
(Figure 2) In general, the molecular ions of those adducts appear with either a very low abundance or absence of the molecular ion along with the fragments of $[\mathrm{M}-29]^{+}$and $\left[\mathrm{XC}_{12} \mathrm{H}_{11}\right]^{+}$as common ions.

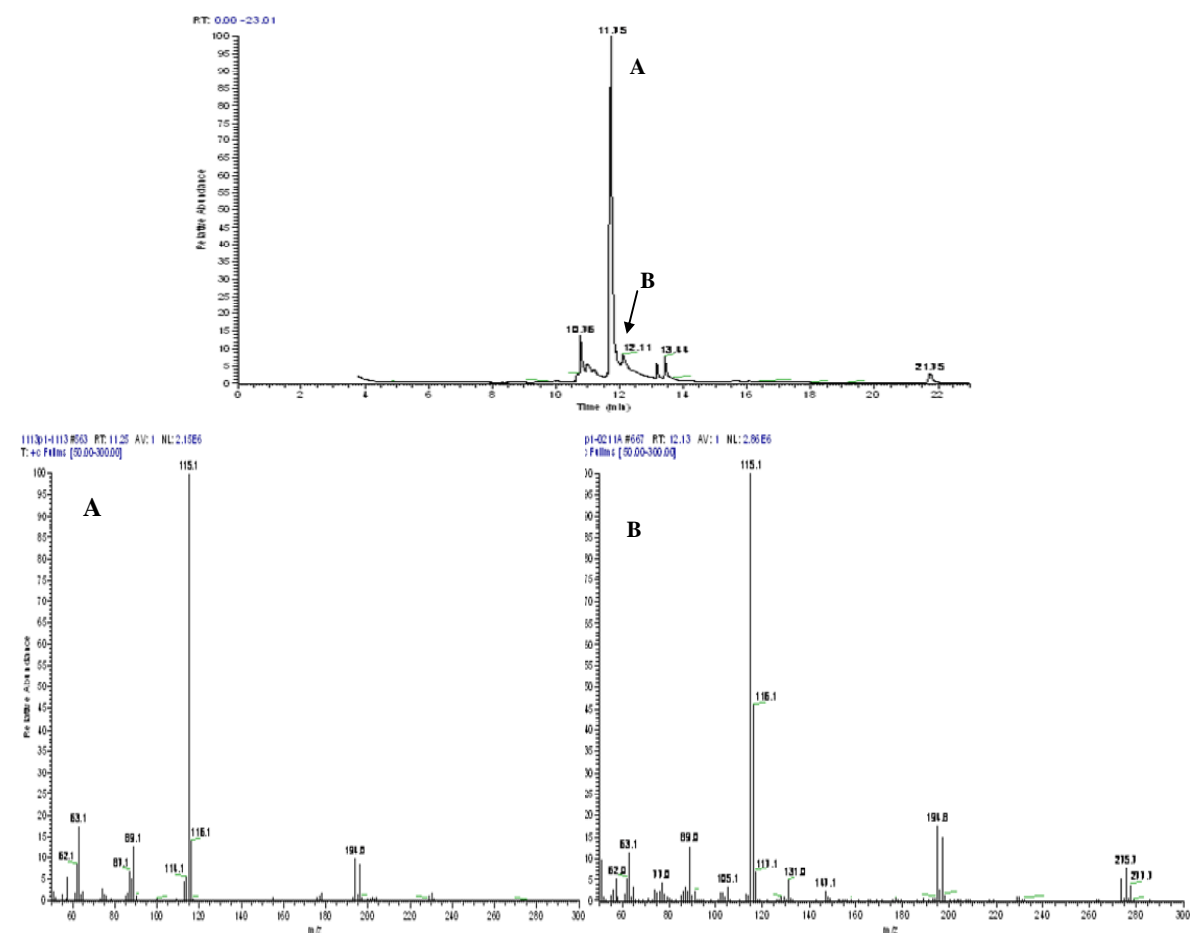

Figure 1. GC-MS analysis of 1-bromo-2-phenylcyclopropene (2a, A) contaminated by trace amounts of 1,1dibromo-2-phenylcyclopropane (B).

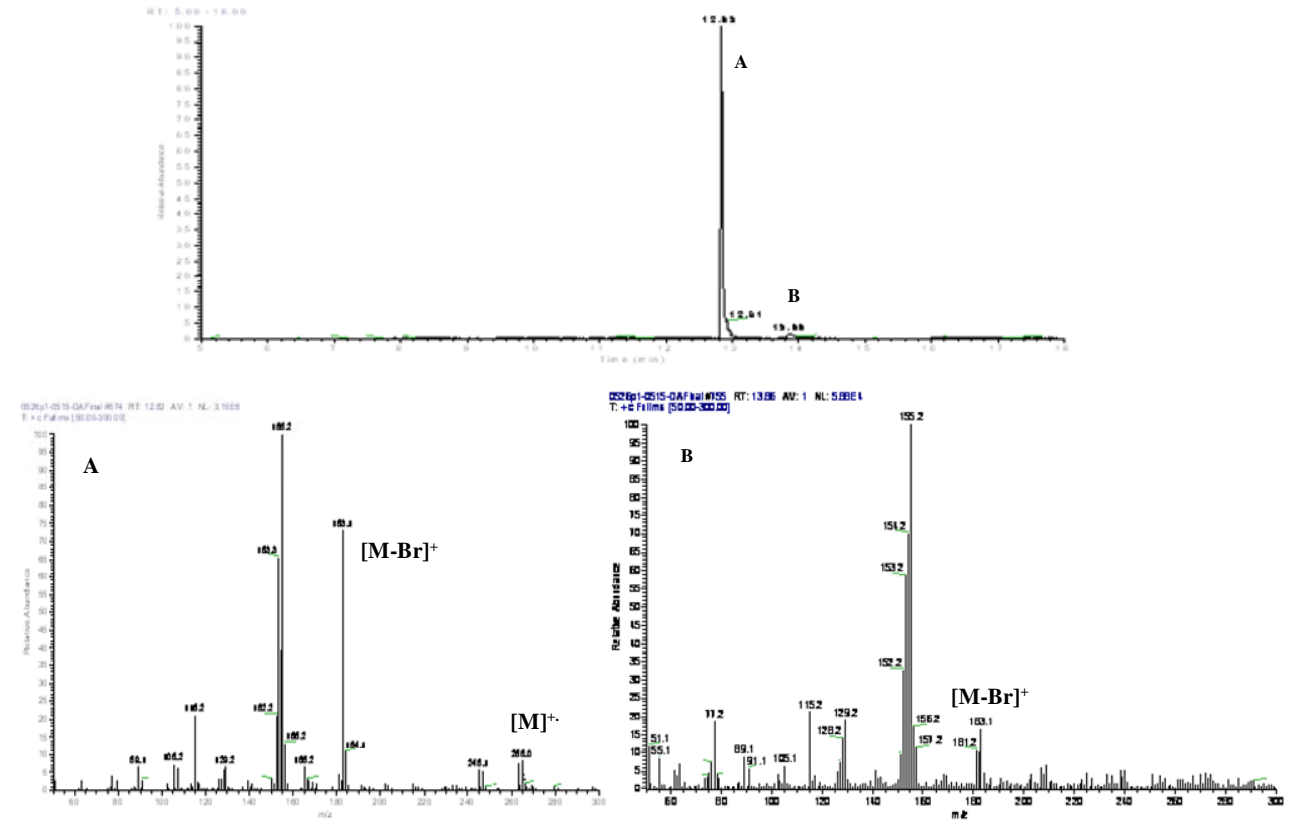

Figure 2. GC-MS analysis of the adduct (4a, A; 6a, B) resulting from addition of 1-bromo-2-phenylcyclopropene and furan. 


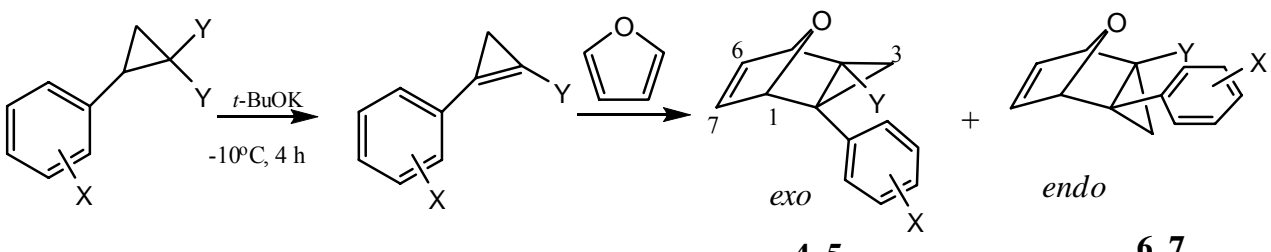

2, 3

4, 5

6, 7

$\mathrm{X}: \mathrm{H},(\mathbf{a}) ; 3-\mathrm{CH}_{3}$ (b); 4- $\mathrm{CH}_{3}$ (c); 3- $\mathrm{OCH}_{3}$ (d); 4-OCH $(\mathbf{-}) ; 3-\mathrm{Cl}$ (f); 4-Cl (g); 3- $\mathrm{Br}(\mathbf{h}) ; 4-\mathrm{Br}(\mathbf{i})$.

$\mathrm{Y}: \mathrm{Br} \mathbf{2}, \mathbf{4}, \mathbf{6} ; \mathrm{Cl}$ 3, 5, 7

Scheme 1

Table 1. Results of the Diels-Alder addition of 1-aryl-2-halocyclopropene and furan in a RTIL ${ }^{\mathrm{a}}$

\begin{tabular}{|c|c|c|c|}
\hline Entry & Cyclopropene/X;Y & Yield $/ \%$ & Ratio exo/endo \\
\hline $1^{\mathrm{b}}$ & $\mathrm{H} ; \mathrm{Br}$ & 7.6 & 90.8 \\
\hline $2^{c}$ & $\mathrm{H} ; \mathrm{Br}$ & 50.6 & 91.2 \\
\hline 3 & $\mathrm{H} ; \mathrm{Br}$ & 54.6 & 89.3 \\
\hline $4^{\mathrm{d}}$ & $\mathrm{H} ; \mathrm{Br}$ & 48.6 & 92.0 \\
\hline $5^{e}$ & $\mathrm{H} ; \mathrm{Br}$ & 54.9 & 95.3 \\
\hline $6^{\mathrm{f}}$ & $\mathrm{H} ; \mathrm{Br}$ & 59.3 & 92.3 \\
\hline 7 & $3-\mathrm{CH}_{3} ; \mathrm{Br}$ & 53.1 & 90.8 \\
\hline 8 & $4-\mathrm{CH}_{3} ; \mathrm{Br}$ & 38.2 & 91.9 \\
\hline 9 & $3-\mathrm{OCH}_{3} ; \mathrm{Br}$ & 46.2 & 90.0 \\
\hline 10 & $4-\mathrm{OCH}_{3} ; \mathrm{Br}$ & 42.3 & 92.4 \\
\hline 11 & $3-\mathrm{Cl} ; \mathrm{Br}$ & 51.9 & 90.1 \\
\hline 12 & 4-Cl; $\mathrm{Br}$ & 51.4 & 92.5 \\
\hline 13 & 3-Br; $\mathrm{Br}$ & 57.3 & 89.2 \\
\hline 14 & 4-Br; $\mathrm{Br}$ & 53.2 & 91.8 \\
\hline 15 & $\mathrm{H} ; \mathrm{Cl}$ & 35.8 & 91.4 \\
\hline 16 & $3-\mathrm{CH}_{3} ; \mathrm{Cl}$ & 37.6 & 90.7 \\
\hline 17 & $4-\mathrm{CH}_{3} ; \mathrm{Cl}$ & 41.2 & 91.8 \\
\hline 18 & $3-\mathrm{OCH}_{3} ; \mathrm{Cl}$ & 40.5 & 89.5 \\
\hline 19 & $4-\mathrm{OCH}_{3} ; \mathrm{Cl}$ & 52.7 & 92.3 \\
\hline 20 & $3-\mathrm{Cl} ; \mathrm{Cl}$ & 51.2 & 90.1 \\
\hline 21 & 4-Cl; $\mathrm{Cl}$ & 49.5 & 90.9 \\
\hline 22 & 3-Br; $\mathrm{Cl}$ & 55.1 & 89.3 \\
\hline 23 & 4-Br; $\mathrm{Cl}$ & 57.1 & 94.3 \\
\hline
\end{tabular}

${ }^{\mathrm{a}}$ The solution $(1.0 \mathrm{~mL})$ of cyclopropene $(2.5 \mathrm{mmol})$ in hexanes and furan $(1.7 \mathrm{~g}, 25 \mathrm{mmol})$ with [omin $]^{+} \mathrm{BF}_{4}^{-}(1.0 \mathrm{~mL}) \mathrm{was}^{-} \mathrm{kept}$ in a glass tube at $30{ }^{\circ} \mathrm{C}$ for $12 \mathrm{~h}$. ${ }^{b}$ Without the presence of a RTIL. ${ }^{\mathrm{c}} 2.0 \mathrm{~mL}$ of [omin] ${ }^{+} \mathrm{BF}_{4}^{-}{ }^{-}$was used. ${ }^{\mathrm{d}}\left[\mathrm{hmmin}^{+} \mathrm{BF}_{4}^{-} \mathrm{was}^{-}\right.$used instead of $[\mathrm{omin}]^{+} \mathrm{BF}_{4}^{-} \cdot{ }^{\mathrm{e}}$ [omin $]^{+} \mathrm{PF}_{6}^{-}$was used instead of $[\mathrm{omin}]^{+} \mathrm{BF}_{4}^{-} \cdot{ }^{-}[\mathrm{hmin}]^{+} \mathrm{PF}_{6}^{-}$was used instead of $[\mathrm{omin}]^{+} \mathrm{BF}_{4}^{-}$.

Due to the low yields of the endo-isomers, only the exo-isomers were isolated for characterization (Table 1). The chemical shifts of $\mathrm{C} 6-\mathrm{H}$ and $\mathrm{C} 7-\mathrm{H}$ and the coupling constant between them were obtained via the treatment according to the non-first order coupling system. ${ }^{1} \mathrm{H}$ NMR spectroscopy appeared to be particularly informative in the compounds 4,5 because one of the cyclopropyl protons appeared consistently at a much lower field than the other. The signal associated with the proton anti to the oxygen atom was found at 1.56 1.63 ppm, while the syn proton appeared between 2.63 and 2.70 ppm. This difference may conceivably be attributed to the diamagnetic anisotropy of the oxygen atom, which causes a decrease in the shielding of the syn proton relative to the anti proton. ${ }^{10}$ This interpretation is in agreement with the formation of the exo isomer at the expense of the endo form due to the lack of a favorable secondary orbital interaction (SOI) in the transition state ${ }^{11}$ as well as the potential congestion between the cyclopropyl proton and the furan ring in the transition state for the formation of the endo-isomer. This result led to a contradiction to the conclusion from the reactions of cyclopropene and furan by Apeloig, "the parent 
cyclopropene and 1,2-disubstituted cyclopropenes are expected to yield endo-adducts exclusively or predominantly. 3,3-Gemdisubstituted cyclopropenes are predicted to yield exo-adducts."

The competition between decomposition and cycloaddition of arylcyclopropenes $\mathbf{2 ,} 3$ and furan resulted in very poor yields of the cycloadducts in common organic solvents or in a solventless system. In this study, we found that the yields of the cycloaddition products can be improved by adding imidazolium type ionic liquids, i.e. 1-hexyl-3-methylimidazolium ([hmin $]^{+}$) and 1-methyl-3-octylimidazolium ([omin $]^{+}$) salts. The reaction rates are also accelerated by the presence of ionic liquids in 50 volume $\%$ or less. However, when more ionic liquids are used, a more difficult chromatographic separation needs to be performed. Normally, the reaction rate can be enhanced by the presence of $25 \mathrm{vol} \%$ of RTIL (entry 2). The distribution of the exo-isomer is not much affected by the used RTIL. The RTIL bearing a longer R group leads to better yields (entries 3, 5 vs. entries 4,6); the type of anion doesn't affect the yields (entries 3, 4 vs. entries 5,6). This different result on the steric selectivity might be due to the bulky aryl ring on the cyclopropene ring creating a congestion effect in the transition state. A less polar cyclopropene leads to a less polar transition state which will poorly interact with a highly polar RTIL.

The extension of this reaction to their gem-difluorocyclopropene $\mathbf{8}$ analogues failed. Although, the compounds 8 are relatively thermally stable and allowed us to carry out the reaction at temperatures higher than $100^{\circ} \mathrm{C}$, this reaction resulted in the recovery of starting material. This might be due to the fact that the cyclopropene ring of compound $\mathbf{8}$ possesses aromaticity. This is deduced from the calculated and observed bond length and dipole moment of the gem-difluorocyclopropene, consistent with the delocalization of electron density via a negative hyperconjugation from the $\pi$-bond into the C-F $\sigma^{*}$ orbital. ${ }^{13}$ Neither pyrrole nor thiophene undergoes the [4+2]-cycloaddition with compound 2.

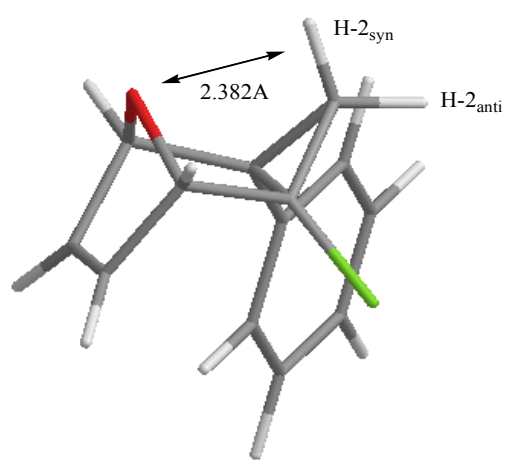

\begin{tabular}{|c|c|c|}
\hline \multirow[t]{2}{*}{ method } & MN2 & AM1/MOPAC \\
\hline & \multicolumn{2}{|c|}{$\begin{array}{l}\text { Distance between oxygen } \\
\text { hydrogen }\left(\mathrm{H}_{2 \text { syn }}\right) \AA\end{array}$} \\
\hline $5 \mathbf{a}$ exo & 2.527 & 2.359 \\
\hline $7 \mathbf{a}$ endo & 2.541 & 2.382 \\
\hline
\end{tabular}

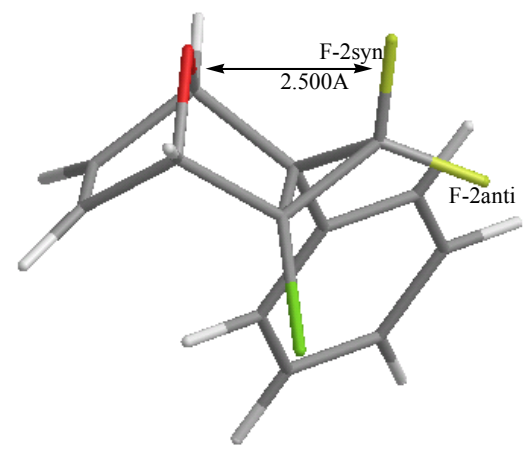

\begin{tabular}{lll}
\hline method & MN2 & \multicolumn{1}{c}{ AM1/MOPAC } \\
\hline & Distance between oxygen and \\
& flourine $\left(\mathrm{F}_{2 \text { syn }}\right) \AA$ \\
8/exo & 2.672 & 2.500 \\
8/endo & 2.703 & 2.537 \\
\hline Sum of van der Waals Radiiof fluorine and oxygen : $2.75 \AA$ \\
\hline
\end{tabular}

Figure 3. Possible distance between oxygen atom and either hydrogen or fluorine atoms obtained from calculation using MN2 and AM1/MOPAC.

The simulated thermal dynamic data from MN2 and AM1/MOPAC are also used to explain the selectivity in this system. The heats of formation of exo- and endo-isomer of $5 \mathbf{a}$ and $7 \mathbf{a}$ are -18.25 and $-12.49 \mathrm{Kcal} / \mathrm{mole}$ with activation energies of 19.55 and $20.55 \mathrm{Kcal} / \mathrm{mole}$, respectively. The distance between the oxygen atom and the hydrogen of the cyclopropane of $5 \mathbf{a}$ and $7 \mathbf{a}$ ranged from 2.359 to $2.527 \AA$ and 2.382 to $2.541 \AA$, respectively, (Figure 1). While the sum of the van der Waal radii of hydrogen and oxygen atoms are ranging from 2.500 to $2.700 \AA .{ }^{14}$ The simulated thermodynamic data suggested that the heat of formation is responsible for the high exo-selectivity. From the same simulation, the results for gem-difluorocyclopropene adduct 8 reveal a heat of formation of -0.588 and $0.65 \mathrm{Kcal} / \mathrm{mole}$ with activation energies of 23.66 and $25.26 \mathrm{Kcal} / \mathrm{mol}$ for exoand endo-isomer respectively, while the distance between the oxygen atom and the fluorine atom range from 2.500 to $2.672 \AA$ 
and 2.537 to $2.703 \AA$ for exo- and endo-adduct, respectively, which are very close to van der Waal's radii ( $2.75 \AA) .{ }^{15}$ The low heat of formation of adduct 8 indicates its instability that is responsible for slowing down the cycloaddition between gemdifluorocyclopropene and furan.

\section{Conclusions}

The thermal unstable compounds1-aryl-2-halocyclopropenes 2, 3 readily undergo cycloaddition with furan in the presence of a RTIL to yield the exo-isomers as the main products. The possible congestion between an aryl ring of cyclopropene and the oxygen of furan might prohibit the formation of the endo-isomer.

\section{Experimental Section}

General. ${ }^{1} \mathrm{H}$ NMR and ${ }^{13} \mathrm{C}$ NMR spectra were recorded at 400, $100 \mathrm{MHz}$ on Bruker Advance-400, respectively, at ambient temperature. Chemical shifts for samples in $\mathrm{CDCl}_{3}$ solution are reported in $\delta$ units relative to TMS $\left({ }^{1} \mathrm{H}\right.$ and $\left.{ }^{13} \mathrm{C}\right)$. Mass spectra were obtained from GC/MS (Fisons 8000 series coupled with Finnigan MD-800) at an ionization potential of $70 \mathrm{eV}$. High resolution mass spectra were determined using a JEOL JMX SX/SX 102A mass spectrometer at the Instrumental Analytic Center at National Chung-Hsing University. IR spectra were recorded of a film on KBr plate with a Perkin-Elmer System 2000 FT-IR spectrometer. Elemental analyses were performed at the Instrumental Analytic Center at National Chung-Hsing University. Melting points were measured on a Yanaco MP-J3 micro melting apparatus and are uncorrected. 2,2Difluorostyrenes were prepared from the corresponding aldehyde and $\mathrm{ClCF}_{2} \mathrm{CO}_{2} \mathrm{Na}$ in the presence of triphenylphosphine in a diglyme solution at $180{ }^{\circ} \mathrm{C} .{ }^{16}$

1-Aryl-2,2-dihalocyclopropanes were prepared from the reaction of styrene and $\mathrm{CHX}_{3}(\mathrm{X}=\mathrm{Br}, \mathrm{Cl})$ in hexanes solution with the presence of $t$-BuOK. ${ }^{17}$ 1-Aryl-2-halocyclopropenes 2,3 were prepared by a dehydrohalogenation of 1-aryl-2,2dihalocyclopropanes using $t$-BuOK at $0{ }^{\circ} \mathrm{C} .{ }^{18}$ Compounds 2, 3 were isolated as a hexanes solution which was used without purification. Their concentration was estimated by GLC analysis. 1-Aryl-3,3-difluoro-2-halocyclopropenes were prepared from 2,2-difluorostyrene, $\mathrm{CHCl}_{3}$, and $\mathrm{NaOH}$ in the presence of a phase transfer reagent. ${ }^{19}$

The structural elucidation of adducts 4, 5 were simplified using 2D NMR experiments (COSY, HSQC, and HMBC), in addition to the standard analysis listed.

\section{Reaction of 1-aryl-2-halocyclopropene and furan. General procedure}

The solution $(1 \mathrm{~mL})$ of 1-bromo-2-phenylcyclopropene $(0.5 \mathrm{~g}, 2.5 \mathrm{mmol})$ in hexanes, prepared from a dehydrohalogenation of 1,1-dibromo-2-phenylcyclopropane, and distilled furan $(1.7 \mathrm{~g}, 25 \mathrm{mmol})$ with [omin] ${ }^{+} \mathrm{BF}_{4}^{-}(1.0 \mathrm{~mL})$ was sealed in a thick glass tube and kept at $30^{\circ} \mathrm{C}$ for $12 \mathrm{~h}$. The resulting reaction mixture was poured into water $(20 \mathrm{~mL})$ and was then extracted with hexanes $(3 \times 15 \mathrm{~mL})$. The organic portion was then dried over $\mathrm{MgSO}_{4}$, filtered and the solvent was removed under reduced pressure, the residue was purified by flash chromatography on a silica gel column to give 2-bromo-4-phenyl-exo-8-oxotricyclo[3.2.10 ${ }^{2,4}$ ]oct-6-ene 4a as a high viscous yellow liquid. ${ }^{1} \mathrm{H}$ NMR $\delta 1.62(\mathrm{~d}, 1 \mathrm{H}, \mathrm{C} 3-\mathrm{H}, J 6.0 \mathrm{~Hz}), 2.71(\mathrm{~d}, 1 \mathrm{H}, \mathrm{C} 3-\mathrm{H}, J$ $6.0 \mathrm{~Hz}$ ), 4.83 (d, 1H, C1-H, J 2.0 Hz), 5.01 (d, 1H, C5-H, J 2.0 Hz), 6.80 (dd, 1H, C6-H, J 6.0, 2.0 Hz), 6.83 (dd, 1H, C7-H, J 6.0, $2.0 \mathrm{~Hz}), 7.27 \sim 7.35$ (m, 5H); ${ }^{13} \mathrm{C}$ NMR $\delta 30.8$ (C3), 39.3 (C4), 48.8 (C2), 81.5 (C5), 82.6 (C1), 127.1(C2'), 128.1(C4'),

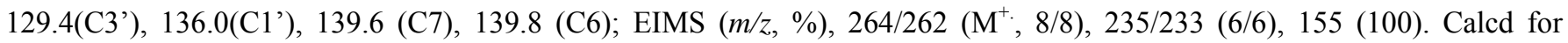
$\mathrm{C}_{13} \mathrm{H}_{11} \mathrm{BrO}$ : C, 59.54; H, 4.23. Found: C, 59.52; H, 4.20; EI-HRMS m/z calcd. For $\mathrm{C}_{13} \mathrm{H}_{11} \mathrm{BrO} 261.9993$; found 261.9997.

2-Bromo-4-(3'-tolyl)-exo-8-oxo-tricyclo[3.2.1.0 ${ }^{2,4}$ ]oct-6-ene (4b). Yellow solid, mp. $60-62{ }^{\circ} \mathrm{C} ;{ }^{1} \mathrm{H}$ NMR $\delta 1.61(\mathrm{~d}, 1 \mathrm{H}, J 6.0$ Hz), 2.36 (s, 3H, $\mathrm{CH}_{3}$ ), 2.68 (d, 1H, C3-H, J $\left.6.0 \mathrm{~Hz}\right), 4.81$ (d, 1H, C1-H, J $\left.2.0 \mathrm{~Hz}\right), 5.01$ (d, 1H, C5-H, J $\left.2.0 \mathrm{~Hz}\right), 6.78$ (dd, $1 \mathrm{H}$, C6-H, J 6.0, $2.0 \mathrm{~Hz}), 6.83(\mathrm{dd}, 1 \mathrm{H}, \mathrm{C} 7-\mathrm{H}, J$ 6.0, $2.0 \mathrm{~Hz}), 7.09 \sim 7.24(\mathrm{~m}, 4 \mathrm{H}) ;{ }^{13} \mathrm{C} \mathrm{NMR} \delta 21.5\left(\mathrm{CH}_{3}\right), 30.7(\mathrm{C} 3), 39.3(\mathrm{C} 4), 48.9$ (C2), 81.5 (C5), 82.6 (C1), 126.4 (C6'), 128.0(C2', 4'), 130.3(C5'), 135.9(C3'), 137.8(C1'), 139.5 (C7), 139.9 (C6); EIMS $(\mathrm{m} / \mathrm{z}, \%)$ 278/276 [( $\left.\mathrm{M}^{+}\right)$6/6], 249/247(3/3), 169(100); Calcd for $\mathrm{C}_{14} \mathrm{H}_{13} \mathrm{BrO}$ : C, 60.86; H, 4.74. Found C, 60.81; H, 4.78; EIHRMS $m / z$ calcd. for $\mathrm{C}_{14} \mathrm{H}_{13} \mathrm{BrO} 276.0150$; found 276.0154.

2-Bromo-4-(4'-tolyl)-exo-8-oxo-tricyclo[3.2.10 ${ }^{2,4}$ ]oct-6-ene (4c). Yellow solid, mp 90-92 ${ }^{\circ} \mathrm{C}$; ${ }^{1} \mathrm{H}$ NMR $\delta 1.62(\mathrm{~d}, 1 \mathrm{H}, \mathrm{C} 3-\mathrm{H}, J$ $6.0 \mathrm{~Hz}), 2.37$ (s, 3H, $\left.\mathrm{CH}_{3}\right), 2.71(\mathrm{~d}, 1 \mathrm{H}, \mathrm{C} 3-\mathrm{H}, J 6.0 \mathrm{~Hz}), 4.83(\mathrm{~d}, 1 \mathrm{H}, \mathrm{C} 1-\mathrm{H}, J 1.0 \mathrm{~Hz}), 5.03(\mathrm{~d}, 1 \mathrm{H}, \mathrm{C} 5-\mathrm{H}, J 1.0 \mathrm{~Hz}), 6.81(\mathrm{dd}$, 1H, C6-H, J 6.0, 2.0 Hz), 6.85 (dd, 1H, C7-H, J 6.0, 2.0 Hz), 7.17 (d, 2H, C3', 5'-H, J 8.0 Hz), 7.26 (d, 2H, C'2, 6'-H, J 8.0 $\mathrm{Hz}) ;{ }^{13} \mathrm{C}$ NMR $\delta 21.3\left(\mathrm{CH}_{3}\right), 30.1$ (C3), 39.0 (C4), 48.9 (C2), 81.5 (C5), 82.5 (C1), 128.8(C2'), 129.3(C3'), 132.9(C4'), 
136.9(C1'), 139(C7), 139.9 (C6); EIMS (m/z, \%) 278/276 [( $\left.\mathrm{M}^{+}\right)$9/9], 249/247 (3/3), 169 (100); Calcd for $\mathrm{C}_{14} \mathrm{H}_{13} \mathrm{BrO}$ : $\mathrm{C}$, 60.86; H, 4.74. Found: C, 60.88; H, 4.71; EI-HRMS m/z calcd. for $\mathrm{C}_{14} \mathrm{H}_{13} \mathrm{BrO} 276.0150$; found 276.0152 .

2-Bromo-4-(3'-anisyl)-exo-8-oxo-tricyclo[3.2.1.0 ${ }^{2,4}$ ]oct-6-ene (4d). Highly viscous yellow liquid; ${ }^{1} \mathrm{H}$ NMR $\delta 1.61$ (d, $1 \mathrm{H}, \mathrm{C} 3-$ $\mathrm{H}, J 6.0 \mathrm{~Hz}), 2.68(\mathrm{~d}, 1 \mathrm{H}, J 6.0 \mathrm{~Hz}), 3.82\left(\mathrm{~s}, 3 \mathrm{H}, \mathrm{OCH}_{3}\right), 4.82(\mathrm{~d}, 1 \mathrm{H}, \mathrm{C} 1-\mathrm{H}, J 1.0 \mathrm{~Hz}), 5.01(\mathrm{~d}, 1 \mathrm{H}, \mathrm{C} 5-\mathrm{H}, J 1.0 \mathrm{~Hz}), 6.82 \sim 6.94$ (m, 4H), 7.16 7.18 (m, 2H); ${ }^{13} \mathrm{C}$ NMR $\delta 30.7(\mathrm{C} 3), 39.1(\mathrm{C} 4), 48.8(\mathrm{C} 2), 55.2\left(\mathrm{OCH}_{3}\right), 81.4(\mathrm{C} 5), 82.6(\mathrm{C} 1), 112.3\left(\mathrm{C} 2{ }^{\prime}\right)$,

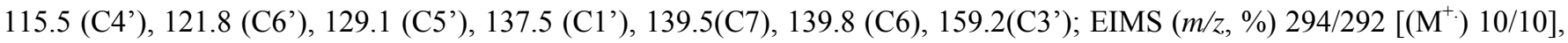
265/ 263(4/4), 185 (100), 170, 145, 115; Calcd for $\mathrm{C}_{14} \mathrm{H}_{13} \mathrm{BrO}_{2}$ : C, 57.53; H, 4.49. Found: C, 57.50; H, 4.51; EI-HRMS m/z calcd. for $\mathrm{C}_{14} \mathrm{H}_{13} \mathrm{BrO}_{2} 292.0099$; found 292.0102 .

2-Bromo-4-(4'-ansiyl)-exo-8-oxo-tricyclo[3.2.1.0 ${ }^{2,4}$ ]oct-6-ene (4e). Light yellow solid, mp $65 \sim 68{ }^{\circ} \mathrm{C}$; ${ }^{1} \mathrm{H}$ NMR $\delta 1.62(\mathrm{~d}, 1 \mathrm{H}$, C3-H, J 6.0 Hz), 2.71 (d, 1H, (C3-H, J $6.0 \mathrm{~Hz}), 3.82\left(\mathrm{~s}, 3 \mathrm{H}, \mathrm{OCH}_{3}\right), 4.76(\mathrm{~d}, 1 \mathrm{H}, \mathrm{C} 1-\mathrm{H}, J 1.0 \mathrm{~Hz}), 4.97$ (d, 1H, (C5-H, J 1.0 $\mathrm{Hz}), 6.78 \sim 6.85(\mathrm{~m}, 2 \mathrm{H}), 6.85 \sim 6.93(\mathrm{~m}, 2 \mathrm{H}), 7.16 \sim 7.18(\mathrm{~m}, 2 \mathrm{H}) ;{ }^{13} \mathrm{C}$ NMR $\delta 30.8(\mathrm{C} 3), 39.6(\mathrm{C} 4), 48.9(\mathrm{C} 2), 55.3\left(\mathrm{OCH}_{3}\right), 81.6$

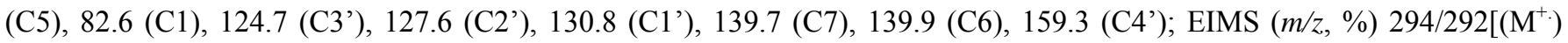
14/14], 265/263 (6/6), 185 (100), 170, 153, 145, 115; Calcd for $\mathrm{C}_{14} \mathrm{H}_{13} \mathrm{BrO}_{2}$ : C, 57.53; H, 4.49. Found: C, 57.59; H, 4.46; EIHRMS $\mathrm{m} / \mathrm{z}$ calcd. for $\mathrm{C}_{14} \mathrm{H}_{13} \mathrm{BrO}_{2} 292.0099$; found 292.0094 .

2-Bromo-4-(3'-chlorophenyl)-exo-8-oxo-tricyclo[3.2.1.0 ${ }^{2,4}$ ]oct-6-ene (4f). Yellow liquid; ${ }^{1} \mathrm{H}$ NMR $\delta 1.60$ (d, 1H, C3-H, J 6.0 Hz), 2.71 (d, 1H, C3-H, J 6.0 Hz), 4.81 (d, 1H, C1-H, J 2.0 Hz), 5.01 (d, 1H, C5-H, J 2.0 Hz), 6.76 (dd, 1H, C6-H, J 6.0 Hz, $2.0 \mathrm{~Hz}$ ), $6.84(\mathrm{dd}, 1 \mathrm{H}, \mathrm{C} 7-\mathrm{H}, J$ 6.0, $2.0 \mathrm{~Hz}), 7.20 \sim 7.33(\mathrm{~m}, 4 \mathrm{H}) ;{ }^{13} \mathrm{C}$ NMR $\delta 30.8(\mathrm{C} 3), 39.1(\mathrm{C} 4), 48.5(\mathrm{C} 2), 81.2(\mathrm{C} 5), 82.5$ (C1), 127.4 (C6’), 127.6(C2'), 129.3(C4'), 129.6(C5'), 133.9 (C6), 138.1(C1'), 139.5(C7), 139.9 (C6); EIMS (m/z, \%) 298/296 [( $\left.\mathrm{M}^{+}\right)$5/5], 269/267 (2/2), 153(100); Calcd for $\mathrm{C}_{13} \mathrm{H}_{10} \mathrm{BrClO}$ : C, 52.71; H, 3.41. found: C, 52.68; H, 3.40; EI-HRMS m/z calcd. for $\mathrm{C}_{13} \mathrm{H}_{10} \mathrm{BrClO} 295.9604$; found 295.9604 .

2-Bromo-4-(4'-chlorophenyl)-exo-8-oxo-tricyclo[3.2.1.0 ${ }^{2,4}$ ]oct-6-ene (4g). Yellow viscous liquid; ${ }^{1} \mathrm{H}$ NMR $\delta 1.56(\mathrm{~d}, 1 \mathrm{H}$, C3-H, J 6.0 Hz), 2.70 (d, 1H, C3-H, J 6.0 Hz), 4.79 (d, 1H, C1-H, J 2.0 Hz), 5.00 (d, 1H, C5-H, J 2.0 Hz), 6.77 (dd, 1H, C6-H, $J$ 6.0, $2.0 \mathrm{~Hz}), 6.84$ (dd, 1H, C7-H, J 6.0, 2.0Hz), 7.24 7.30 (m, 2H), 7.31 7.41 (m, 2H); ${ }^{13} \mathrm{C} \delta$ NMR 30.8 (C3), 38.9 (C4), 48.6 (C2), 81.4 (C5) 82.5 (C1), 128.3 (C2'), 130.9 (C3’), 133.3 (C4'), 134.6(C1'), 139.5 (C7), 139.9 (C6); EIMS (m/z, \%) 298/296 [( $\left.\mathrm{M}^{+}\right)$5/5], 269/267 (3/3), 153 (100); Calcd for $\mathrm{C}_{13} \mathrm{H}_{10} \mathrm{BrClO}$ : C, 52.71; H, 3.41. Found: C, 52.70; H, 3.41; EI-HRMS m/z calcd. for $\mathrm{C}_{13} \mathrm{H}_{10} \mathrm{BrClO} 295.9604$; found 295.9607 .

2-Bromo-4-(3'-bromophenyl)-exo-8-oxo-tricyclo[3.2.1.0,4 ${ }^{2,4}$ oct-6-ene (4h). Yellow viscous liquid; ${ }^{1} \mathrm{H}$ NMR $\delta 1.61$ (d, $1 \mathrm{H}$, C3-H, J 6.0 Hz), 2.71 (d, 1H, C3-H, J $\left.6.0{ }^{1} \mathrm{~Hz}\right), 4.80$ (d, 1H, C1-H, J $\left.2.0 \mathrm{~Hz}\right), 5.01$ (d, 1H, C5-H, J 2.0 Hz), 6.78 (dd, 1H, C6$\mathrm{H}, J$ 6.0, $2.0 \mathrm{~Hz}), 6.85(\mathrm{dd}, 1 \mathrm{H}, \mathrm{C} 7-\mathrm{H}, J 6.0,2.0 \mathrm{~Hz}), 7.17 \sim 7.47(\mathrm{~m}, 4 \mathrm{H}) ;{ }^{13} \mathrm{C}$ NMR $\delta 30.8(\mathrm{C} 3), 39.1(\mathrm{C} 4), 48.5(\mathrm{C} 2), 81.3$ (C5), 82.6 (C1), 122.1 (C3’), 128.1(C6’), 129.7(C4'), 130.4(C2'), 132.6 (C5'), 138.5 (C1'), 139.5 (C7), 139.9 (C6); EIMS $(\mathrm{m} / \mathrm{z}, \%)$ 313/311 [(M-29) $\left.{ }^{+}, 4 / 4\right], 153$ (100); Calcd for $\mathrm{C}_{13} \mathrm{H}_{10} \mathrm{Br}_{2} \mathrm{O}$ : C, 45.89; H, 2.96. Found: $\mathrm{C}, 45.90 ; \mathrm{H}, 2.97$; EI-HRMS m/z calcd. for $\left[\mathrm{C}_{13} \mathrm{H}_{10} \mathrm{Br}_{2} \mathrm{O}-\mathrm{CHO}\right], 310.9071$; found 310.9072 .

2-Bromo-4-(4'-bromophenyl)-exo-8-oxo-tricyclo[3.2.1.0,4 ${ }^{2,4}$ oct-6-ene (4i). Yellow viscous liquid; ${ }^{1} \mathrm{H}$ NMR $\delta 1.56(\mathrm{~d}, 1 \mathrm{H}$, C3-H, J 6.0 Hz), 2.71 (d, 1H, C3-H, J 6.0 Hz), 4.79 (d, 1H, C1-H, J 2.0 Hz), 5.00 (d, 1H, C5-H, J 2.0 Hz), 6.76 (dd, 1H, C6-H, $J$ 6.0, 2.0 Hz), 6.84 (dd, 1H, C7-H, J 6.0, 2.0 Hz), 7.20 (d, 2H, C2',6'-H, J 8.0 Hz), 7.44 (d, 2H, C3', 5'-H, J 8.0 Hz); ${ }^{13} \mathrm{C}$ NMR \& 30.8 (C3), 38.9 (C4), 48.6 (C2), 81.3 (C5), 82.5 (C1), 121.5 (C4'), 131.3 (C2'), 131.7 (C3'), 135.1 (C1'), 139.5 (C7), 139.9 (C6); EIMS (m/z, \%) 313/311 [(M-29) $\left.{ }^{+}, 6 / 6\right], 153$ (100); Calcd for $\mathrm{C}_{13} \mathrm{H}_{10} \mathrm{Br}_{2} \mathrm{O}$ : C, 45.89; H, 2.96. Found:C, 45.92; H, 2.95; EIHRMS $m / z$ calcd. for $\left[\mathrm{C}_{13} \mathrm{H}_{10} \mathrm{Br}_{2} \mathrm{O}-\mathrm{CHO}\right] 310.9071$; found 310.9070 .

2-Chloro-4-phenyl-exo-8-oxo-tricyclo[3.2.1.0 ${ }^{2,4}$ ]oct-6-ene (5a). Yellow viscous liquid; ${ }^{1} \mathrm{H}$ NMR $\delta 1.61(\mathrm{~d}, 1 \mathrm{H}, \mathrm{C} 3-\mathrm{H}, J 6.0$ Hz), 2.64 (d, 1H, C3-H, J 6.0 Hz), 4.88 (d, 1H, C1-H, J 2.0 Hz), 4.97 (d, 1H, C5-H, J 2.0 Hz), 6.83 (dd, 1H, C6-H, J 6.0, 2.0 $\mathrm{Hz}), 6.87$ (dd, 1H, C5-H, J 6.0, 2.0 Hz), 7.19 7.32 (m, 5H); ${ }^{13} \mathrm{C}$ NMR $\delta 29.7$ (C3), 39.8 (C4), 58.0 (C2), 81.6 (C5), 82.0 (C1), 127.2 (C2'), 128.1 (C4'), 129.3 (C3'), 135.6 (C1'), 139.1 (C7), 140.2 (C6); EIMS (m/z, \%) 220/218 [((M+) 7/21], 191/189 (2/7), 155 (100); Calcd for $\mathrm{C}_{13} \mathrm{H}_{11} \mathrm{ClO}$ : C, 71.54; H, 5.08. Found: C, 71.57; H, 5.09; EI-HRMS m/z calcd. for $\mathrm{C}_{13} \mathrm{H}_{11} \mathrm{ClO}$ 218.0498; found 218.0495.

2-Chloro-4-(3'-tolyl)-exo-8-oxo-tricyclo[3.2.1.0 ${ }^{2,4}$ ]oct-6-ene (5b). Yellow viscous liquid; ${ }^{1} \mathrm{H}$ NMR $\delta 1.63$ (d, $1 \mathrm{H}, \mathrm{C} 3-\mathrm{H}, J$ $6.0 \mathrm{~Hz}$ ), 2.36 (s, 3H, CH $\mathrm{CH}_{3}, 2.66$ (d, 1H, C3-H, J $\left.6.0 \mathrm{~Hz}\right), 4.90$ (d, 1H, C1-H, J $\left.2.0 \mathrm{~Hz}\right), 5.00$ (d, 1H, C5-H, J 2.0 Hz), 6.86 (dd, 1H, C6-H, J 6.0, $2.0 \mathrm{~Hz}), 6.88$ (dd, 1H, C7-H, J 6.0, $2.0 \mathrm{~Hz}), 7.13 \sim 7.23(\mathrm{~m}, 4 \mathrm{H}) ;{ }^{13} \mathrm{C}$ NMR $\delta 21.4\left(\mathrm{CH}_{3}\right), 29.7$ (C3), 39.7 (C4), 58.0 (C2), 81.6 (C5), 81.9 (C1), 126.3 (C6'), 128.0 (C4'), 128.5 (C2'), 130.1 (C5'), 135.4 (C3'), 137.7 (C1'), 138.9(C7), 140.2 (C6); EIMS ( $\mathrm{m} / \mathrm{z}, \%)$ 234/.232 [( $\left.\mathrm{M}^{+}\right)$2/7], 205/203 (33/100); Calcd for $\mathrm{C}_{14} \mathrm{H}_{13} \mathrm{ClO}$ : C, 72.39; H, 5.65. Found: C, 72.38; H, 5.67; EI-HRMS $m / z$ calcd. for $\mathrm{C}_{14} \mathrm{H}_{13} \mathrm{ClO} 232.0655$; found 232.0653 . 
2-Chloro-4-(4-tolyl)-exo-8-oxo-tricyclo[3.2.1.0 ${ }^{2,4}$ ]oct-6-ene (5c). Yellow viscous liquid; ${ }^{1} \mathrm{H}$ NMR $\delta 1.61(\mathrm{~d}, 1 \mathrm{H}, \mathrm{C} 3-\mathrm{H}, \mathrm{J} 6.0$ $\mathrm{Hz}), 2.36\left(\mathrm{~s}, 3 \mathrm{H}, \mathrm{CH}_{3}\right), 2.64$ (d, 1H, C3-H, J 6.0 Hz), 4.87 (d, 1H, C1-H, J 1.0 Hz), 4.97 (d, 1H, C5-H, J 1.0 Hz), 6.83 (dd, 1H, C6-H, J 6.0, $2.0 \mathrm{~Hz}), 6.87(\mathrm{dd}, 1 \mathrm{H}, \mathrm{C} 7-\mathrm{H}, J 6.0,2.0 \mathrm{~Hz}), 7.08 \sim 7.17(\mathrm{~m}, 2 \mathrm{H}), 7.20 \sim 7.35(\mathrm{~m}, 2 \mathrm{H}) ;{ }^{13} \mathrm{C}$ NMR $\delta 21.4\left(\mathrm{CH}_{3}\right), 29.7$ (C3), 39.5 (C4), 57.9 (C2), 82.0 (C5), 81.6 (C1), 128.9 (C2'), 129.3 (C3'), 132.4 (C4'), 136.9 (C1'), 138.9 (C6), 140.3 (C7); EIMS (m/z, \%) 234/232 [( $\left(\mathrm{M}^{+}\right)$5/16], 205/203 (33/100); Calcd for $\mathrm{C}_{14} \mathrm{H}_{13} \mathrm{ClO}$ : C, 72.39; H, 5.65. Found: C, 72.40; H, 5.65; EIHRMS $m / z$ calcd. for $\mathrm{C}_{14} \mathrm{H}_{13} \mathrm{ClO} 232.0655$; found 232.0655 .

2-Chloro-4-(3'-anisyl)-exo-8-oxo-tricyclo[3.2.1.0 ${ }^{2,4}$ ]oct-6-ene (5d). White solid, mp $68 \sim 70{ }^{\circ} \mathrm{C} ;{ }^{1} \mathrm{H}$ NMR $\delta 1.61(\mathrm{~d}, 1 \mathrm{H}, \mathrm{C} 3-\mathrm{H}$, $J 6.0 \mathrm{~Hz}), 2.64(\mathrm{~d}, 1 \mathrm{H}, \mathrm{C} 3-\mathrm{H}, J 6.0 \mathrm{~Hz}), 3.82\left(\mathrm{~s}, 3 \mathrm{H}, \mathrm{OCH}_{3}\right), 4.86(\mathrm{~d}, 1 \mathrm{H}, \mathrm{C} 1-\mathrm{H}, J 1.0 \mathrm{~Hz}), 4.95(\mathrm{~d}, 1 \mathrm{H}, \mathrm{C} 5-\mathrm{H}, J 1.0 \mathrm{~Hz})$, 6.82 6.94 (m, 5H), 7.24 7.28 (m, 1H); ${ }^{13} \mathrm{C}$ NMR $\delta 29.8(\mathrm{C} 3), 39.8(\mathrm{C} 4), 55.2\left(\mathrm{OCH}_{3}\right), 58.0(\mathrm{C} 2), 81.7(\mathrm{C} 5), 82.0(\mathrm{C} 1), 112.3$

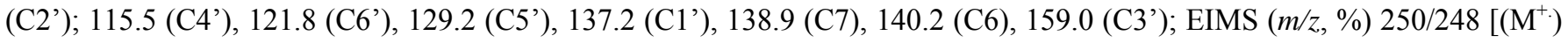
7/21], 221/219 (100); Calcd for $\mathrm{C}_{14} \mathrm{H}_{13} \mathrm{ClO}_{2}: \mathrm{C}, 67.73 ; \mathrm{H}, 5.28$. Found: $\mathrm{C}, 67.76 ; \mathrm{H}, 5.29$; EIHRMS m/z calcd. for $\mathrm{C}_{14} \mathrm{H}_{13} \mathrm{ClO}_{2}$ 248.0604; found 248.0602 .

2-Chloro-4-(4'-anisyl)-exo-8-oxo-tricyclo[3.2.1.0 ${ }^{2,4}$ ]oct-6-ene (5e). Light yellow solid, mp $132 \sim 134{ }^{\circ} \mathrm{C} ;{ }^{1} \mathrm{H}$ NMR $\delta 1.61(\mathrm{~d}$, 1H, C3-H, J 6.0 Hz), 2.64 (d, 1H, C3-H, J 6.0 Hz), 4.85 (d, 1H, C1-H, J 1.0 Hz), 4.95 (d, 1H, C5-H, J 1.0 Hz), $6.78 \sim 6.85$ (m, 2H), 6.85 6.93 (m, 2H), 7.16 7.24(m, 2H); ${ }^{13} \mathrm{C}$ NMR $\delta 29.7(\mathrm{C} 3), 39.2(\mathrm{C} 4), 55.4\left(\mathrm{OCH}_{3}\right), 57.6(\mathrm{C} 2), 81.6(\mathrm{C} 5), 82.2(\mathrm{C} 1)$,

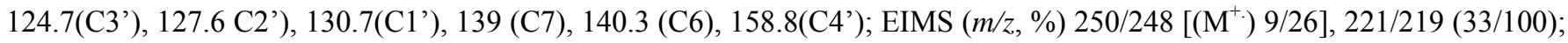
Calcd for $\mathrm{C}_{14} \mathrm{H}_{13} \mathrm{ClO}_{2}$ : C, 67.73; H, 5.28. found: C, 67.72; H, 5.30; EIHRMS m/z calcd. for $\mathrm{C}_{14} \mathrm{H}_{13} \mathrm{ClO}_{2}$ 248.0604; found 248.0601.

2-Chloro-4-(3'-chlorophenyl)-exo-8-oxo-tricyclo[3.2.1.0 ${ }^{2,4}$ ]oct-6-ene (5f). Yellow viscous liquid; ${ }^{1} \mathrm{H}$ NMR $\delta 1.57$ (d, $1 \mathrm{H}$, C3-H, J 6.0 Hz), 2.63 (d, 1H, C3-H, J 6.0 Hz), 4.85 (d, 1H, C1-H, J 2.0 Hz), 4.96 (d, 1H, C5-H, J 2.0 Hz), 6.79 (dd, 1H, C6-H, $J$ 6.0, $2.0 \mathrm{~Hz}), 6.87$ (dd, 1H, C7-H, J 6.0, $2.0 \mathrm{~Hz}), 7.18 \sim 7.30$ (m, 4H); ${ }^{13} \mathrm{C}$ NMR $\delta 29.8(\mathrm{C} 3), 39.6(\mathrm{C} 4), 57.9(\mathrm{C} 2), 81.6$ (C5), 81.8 (C1), 127.4 (C6'), 127.6 (C2'), 129.2 (C4'), 129.9 (C5'), 134.0 (C3'), 137.7 (C1'), 139.4 (C7), 139.9 (C6); EIMS (m/z, \%) 254/252 [( $\left(\mathrm{M}^{+}\right)$5/9], 227/225/223 (3/18/30), 52 (100); Calcd for $\mathrm{C}_{13} \mathrm{H}_{10} \mathrm{Cl}_{2} \mathrm{O}: \mathrm{C}, 61.90$; H, 4.00. Found: C, 61.92; H, 4.01; EIHRMS $\mathrm{m} / \mathrm{z}$ calcd. for $\mathrm{C}_{13} \mathrm{H}_{10} \mathrm{Cl}_{2} \mathrm{O} 252.0109$; found 252.0109 .

2-Chloro-4-(4'-chlorophenyl)-exo-8-oxo-tricyclo[3.2.1.0 ${ }^{2,4}$ ]oct-6-ene (5g). Yellow viscous liquid; ${ }^{1} \mathrm{H}$ NMR $\delta 1.56(\mathrm{~d}, 1 \mathrm{H}$, C3-H, J 6.0 Hz), 2.63 (d, 1H, C3-H, J 6.0 Hz), 4.85 (d, 1H, C1-H, J 2.0 Hz), 4.97 (d, 1H, C5-H, J 2.0 Hz), 6.78 (dd, 1H, C6-H, $J$ 6.0, $2.0 \mathrm{~Hz}), 6.86$ (dd, 1H, C7-H, J 6.0, $2.0 \mathrm{~Hz}), 7.24 \sim 7.34$ (m, 4H); ${ }^{13} \mathrm{C}$ NMR $\delta 29.8(\mathrm{C} 3), 39.3(\mathrm{C} 4), 57.9$ (C2), 81.6 (C5),

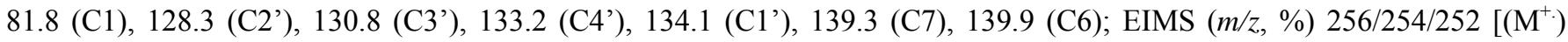
2/11/19)]; 227/225/223 (4/22/41), 152 (100); Calcd for $\mathrm{C}_{13} \mathrm{H}_{10} \mathrm{Cl}_{2} \mathrm{O}$ : C, 61.90; H, 4.00. Found: C, 61.94; H, 4.03; EI-HRMS $\mathrm{m} / \mathrm{z}$ calcd. for $\mathrm{C}_{13} \mathrm{H}_{10} \mathrm{Cl}_{2} \mathrm{O} 252.0109$; found 252.0106.

2-Chloro-4-(3'-bromophenyl)-exo-8-oxo-tricyclo[3.2.1.0 ${ }^{2,4}$ ]oct-6-ene (5h). Yellow viscous liquid; ${ }^{1} \mathrm{H}$ NMR $\delta 1.59$ (d, $1 \mathrm{H}$, C3-H, J 6.0 Hz), 2.65 (d, 1H, C3-H, J 6.0 Hz), 4.84 (d, 1H, C1-H, J 2.0 Hz), 4.97 (d, 1H, C5-H, J 2.0 Hz), 6.77 (dd, 1H, C6-H, $J$ 6.0, $2.0 \mathrm{~Hz}$ ), 6.86 (dd, 1H, C7-H, J 6.0, $2.0 \mathrm{~Hz}), 7.17 \sim 7.50$ (m, 4H); ${ }^{13} \mathrm{C}$ NMR $\delta 29.7$ (C3), $39.4(\mathrm{C} 4), 57.8$ (C2), 81.5 (C5), 81.7 (C1), 122,1(C3'), 127.9 (C6'), 129.6 (C4'), 130.2 (C2'), 132.4 (C5'), 138.0 (C1'), 139.2 (C7), 139.8 (C6); EIMS (m/z, \%) 271/269/267 [(M-29) ${ }^{+}$9/32/34], 188 (100); Calcd for $\mathrm{C}_{13} \mathrm{H}_{10} \mathrm{BrClO}$ : C, 52.71; H, 3.40. Found: C, 52.69; H, 3.41; EI-HRMS m/z calcd. For $\left(\mathrm{C}_{13} \mathrm{H}_{10} \mathrm{BrClO}-\mathrm{CHO}\right) 266.9576$; found 266.9577.

2-Chloro-4-(4'-bromophenyl)-exo-8-oxo-tricyclo[3.2.1.0 ${ }^{2,4}$ ]oct-6-ene (5i). Yellow viscous liquid; ${ }^{1} \mathrm{H}$ NMR $\delta 1.59$ (d, $1 \mathrm{H}$, C3-H, J 6.0 Hz), 2.63 (d, 1H, C3-H, J 6.0 Hz), 4.86 (d, 1H, C1-H, J 2.0 Hz), 4.96 (d, 1H, C5-H, J 2.0 Hz), 6.81 (dd, 1H, C6-H, $J$ 6.0, $2.0 \mathrm{~Hz}), 6.87$ (dd, 1H, J 6.0, $2.0 \mathrm{~Hz}), 7.18 \sim 7.20$ (m, 2H), 7.52 7.57 (m, 2H); ${ }^{13} \mathrm{C}$ NMR $\delta 29.8(\mathrm{C} 3), 39.4(\mathrm{C} 4), 57.9$ (C2), 81.6 (C5), 81.8 (C1), 121.4 (C4'), 128.8 (C2',6'), 131.2 (C3',5'), 134.7(C1'), 139.4 (C7), 139.9 (C6); EIMS (m/z, \%) 271/269/267 [(M-29) ${ }^{+}$14/42/45], 188 (100\%); Calcd for $\mathrm{C}_{13} \mathrm{H}_{10} \mathrm{BrClO}$ : C, 52.71; H, 3.39. Found: C, 52.75; H, 3.42; EI-HRMS $\mathrm{m} / \mathrm{z}$ calcd. for $\left(\mathrm{C}_{13} \mathrm{H}_{10} \mathrm{BrClO}-\mathrm{CHO}\right) 266.9576 ; 266.9576$.

\section{Reaction with pyrrole or thiophene}

The solution $(2.0 \mathrm{~mL})$ of 1-bromo-2- phenylcyclopropene $(0.5 \mathrm{~g}, 2.5 \mathrm{mmol})$ in hexanes, prepared from dehydrohalogenation of 1,1-dibromo-2-phenylcyclopropane, and pyrrole or thiophene $(25 \mathrm{mmol})$ with [omin] ${ }^{+} \mathrm{BF}_{4}^{-}(1.0 \mathrm{~mL})$ was sealed in a thick glass tube and stand at $30{ }^{\circ} \mathrm{C}$ for $12 \mathrm{~h}$. The resultant was poured into water $(20 \mathrm{~mL})$ and then extracted with hexanes $(3 \times 15 \mathrm{~mL})$. GC-MS analyses indicated that two compounds were 1-(3-bromoprop-1-ynyl)benzene and 1-(1-bromopropa-1,2dienyl)benzene in a $2: 3$ ratio. Efforts to isolate an adduct was unsuccessful.

Reaction of 1-bromo-3,3-difluoro-2-phenylcyclopropene (8) with furan 
The mixture of 1-bromo-3,3-difluoro-2-phenylcyclopropene ( $0.58 \mathrm{~g}, 2.5 \mathrm{mmol})$, furan $(1.7 \mathrm{~g}, 25 \mathrm{mmol})$ and [omin] ${ }^{+} \mathrm{BF}_{4}^{-}(2.0$ $\mathrm{mL})$ was sealed in a thick glass tube and kept at $100^{\circ} \mathrm{C}$ for $12 \mathrm{~h}$. The resulting mixture was extracted with hexanes $(3 \times 5 \mathrm{~mL})$. GC-MS analyses indicated the recovery of compound 8.

\section{Computational details}

All of the calculations described above were performed using Gaussian $98 .{ }^{20}$ Computations were carried out at the restricted Hartree-Fock (RHF), ${ }^{21}$ and Density Functional Theory (DFT). DFT calculations used the hybrid B3LYP functional and triple zeta 6-311++G** basis sets. ${ }^{22}$ MN2 and AM1/MOPAC were simulated by using Chem Office 2004, Chem 3D ultra 8.0 ed..

\section{Acknowledgements}

Financial support by the National Science Council of the Republic of China (NSC 90-2113-M-126-007) and technique information support by HSCC are gratefully acknowledged.

\section{References}

1. (a) Liebman J. F.; Greenberg A. Chem. Rev. 1976, 76, 311. (b) Billups W. E.; Haley M. M.; Lee G. -A. Chem. Rev. 1989, 89, 1147. (c) Halton B.; Banwell M. B. Cyclopropenes. In The Chemistry of the Cyclopropyl Group; Rappoport, Z., Ed; Wiley: New York, 1987; Chapter 21.

2. Wiberg K. B. Structures, Energies and Spectra of Cyclopropanes. In The Chemistry of the Cyclopropyl Group; Rappoport, Z., Ed.; Wiely: New York, 1987; Chapter 1.

3. (a) Stigliani W. M.; Laurie V. W.; Li J. C. J. Chem. Phys. 1975, 62, 1890. (b) Kasai P. H.; Myers R. J.; Eggers D. F. Jr.; Wiberg K. B. J. Chem. Phys. 1959, 30, 512. (c) Dunitz J. D.; Feldman H. G.; Schomaker V. J. Chem. Phys. 1952, $20,1708$.

4. Demjanov N. Y.; Doyarenko M. N. Bull. Acad. Sci. USSR. 1922, 16, 297.

5. (a) Alnes K. F. S.; Sydnes L. K. Monatshe. Chem. 2006, 137, 483. (b) Lee G. -A.; Chang C.-Y.; Cherg C. -H.; Chen C. S.; Liu M. J. Chin. Chem. Soc. 2004, 51, 839. (c) Lee G. -A.; Chang C. -Y. Tetrahedron Lett. 1998, 39, 3013. (d) Lee, G. A.; Huang, A. N.; Chen, C. -S.; Li, Y. C.; Jann, Y. -C. J. Org. Chem. 1997, 62, 3355. (e) Binger P.; Wedemann P.; Goddard R.; Brinker U. H. J. Org. Chem. 1996, 61, 6462.

6. Kappe C. O.; Murphree S. S.; Padwa A. Tetrahedron 1997, 53, 14159.

7. Kumar A.; Pawer S. S. J. Org. Chem. 2004, 69, 1419.

8. Fischer, T.; Sethi A.; Welton T.; Woolf J. Tetrahedron Lett. 1999, 40, 793.

9. Berson J. A.; Hamlet Z.; Mueller W. A. J. Am. Chem. Soc. 1962, 84, 297.

10. Baird M. S. Chem. Rev. 2003, 103, 1271.

11. Jackman L. M.; Sternhell S. Applications of Nuclear Magnetic Resonance Spectroscopy in Organic Chemistry, 2nd Edn., Pergaman Press: Oxford; 1969, p 80.

12. Binger P.; Wedemann P.; Goddard R.; Brinker, U. H. J. Org. Chem. 1996, 61, 6462.

13. Apeloig Y.; Arad D.; Kapon M.; Wallerstein M. Tetrahedron Lett. 1987, 28, 5917.

14. (a) Getty S. J.; Hrovat D. A.; Xu J. D.; Barker S. A.; Borden W. T. J. Chem. Soc., Faraday Trans. 1994, 90, 1689. (b) Koppel I. A.; Pihl V.; Koppel J.; Anvia F.; and Taft R. W. J. Am. Chem. Soc. 1994, 116, 8654. (c) Raabe G.; Gais H. -J.; Fleischhauser J. J. Am. Chem. Soc. 1996, 118, 4622. (d) Borden W. T. Chem. Commun. 1998, 1919.

15. Xidos J. D.; Gosse T. L.; Burke E. D.; Poirier R. A.; Burnell D. J. J. Am. Chem. Soc. 2001, 123, 5482.

16. Fuqua S. A.; Duncan W. G.; Silverstein R. M. J. Org. Chem. 1965, 30, 1027.

17. (a) Lin S. T.; Yao Y. F. J. Chin. Chem. Soc. 1992, 39, 415. (b) Henseling K. O. G.; Weyerstahl P. Chem. Ber. 1975, 108, 2804.

18. Wittig G.; Schöllkopf U. Chem. Ber. 1954, 87, 1318.

19. (a) Lin S. T.; Lee C. C. Synthesis 2000, 496. (b) Lin S.T.; Chen L. C. and Lee C. J. J. Chem. Res. 2004, 353-355.

20. Frisch M. J.; et. al. Gaussian Inc.: Pittsburg, PA, 1998.

21. Roothaan C. C. J. Rev. Mod. Phys. 1951, 23, 69.

22. (a) Lee C. Y. W.; Parr R. G. Phys. Rev. B 1988, 37, 785. (b) Becke A. D. J. Chem. Phys. 1993, 98, 5648. 